\title{
Linear equations over multiplicative groups, recurrences, and mixing III
}

H. Derksen, D. Masser

Departement Mathematik und Informatik

Fachbereich Mathematik

Preprint No. 2016-26

November 2016

Universität Basel

CH-4051 Basel

www.math.unibas.ch 
Linear equations over multiplicative groups, recurrences, and mixing III

H. Derksen and D. Masser

\begin{abstract}
Given an algebraic $\mathbf{Z}^{d}$-action corresponding to a prime ideal of a Laurent ring of polynomials in several variables, we show how to find the smallest order $n+1$ of non-mixing. It is known that this is determined by the non-mixing sets of size $n+1$, and we show how to find these in an effective way. When the underlying characteristic is positive and $n \geq 2$, we prove that there are at most finitely many classes under a natural equivalence relation. We work out two examples, the first with 5 classes and the second with 134 classes.
\end{abstract}

2010 MSC codes. 14G17, 37A25

1. Introduction. Not long ago the second author published a paper $[\mathrm{M}]$ about linear equations over multiplicative groups in positive characteristic. This was specifically aimed at an application to a problem about mixing for dynamical systems of algebraic origin, and as a result about linear equations it lacked some of the simplicity of the classical results in zero characteristic. A new feature was the appearance of $n-1$ independently operating Frobenius maps; here $n$ is the number of variables.

Soon afterwards the first author published a paper [D] about recurrences in positive characteristic. He proved an analogue of the Skolem-Lech-Mahler Theorem famous in zero characteristic. A new feature was the appearance of integer sequences involving combinations of $d-2$ powers of the characteristic; here $d$ is the order of the recurrence.

It turns out that these two new features are identical. In positive characteristic the vanishing of a recurrence with $d$ terms can be regarded as a linear equation in $d-1$ variables to be solved in a multiplicative group (so in particular $n-1=d-2$ ). This observation can be developed in three directions.

In Part I of this series [DM1] we gave an improved version of the result of $[\mathrm{M}]$ in a form more closely related to that in zero characteristic. In Part II [DM2] we applied this to recover the result of $[\mathrm{D}]$, and indeed we generalized it to sums of recurrences. Here in Part III we present some new applications to mixing problems for dynamical systems of algebraic origin. In an earlier version we gave an effective algorithm to determine the 
smallest order $n+1$ of non-mixing of any basic action associated with a given prime ideal in a Laurent polynomial ring. This solved the problem (3) mentioned by Klaus Schmidt in $[\mathrm{S}](\mathrm{p} .283)$.

Thanks to the work of $[\mathrm{M}]$ we know that this non-mixing comes from sets of cardinality $n+1$ which are themselves non-mixing for $\alpha$ (see later for definitions). After receiving our solution mentioned above, Klaus Schmidt in a message dated 12th July 2006 asked us if it is possible to determine all these non-mixing sets (or "shapes") effectively. This we do in the present paper, which also includes a different method of determining $n$.

For a positive integer $d$ let $\alpha$ be a $\mathbf{Z}^{d}$-action on a compact abelian group. We have three possibilities:

(I) there is $n$-mixing but not $(n+1)$-mixing for some unique $n=n(\alpha) \geq 2$,

(II) there is no 2-mixing,

(III) there is $n$-mixing for all $n \geq 2$.

In case (II) we may write $n(\alpha)=1$, and in case (III) we may write $n(\alpha)=\infty$.

Write $\mathcal{R}=\mathcal{R}_{d}$ for the Laurent polynomial ring $\mathbf{Z}\left[u_{1}, u_{1}^{-1}, \ldots, u_{d}, u_{d}^{-1}\right]$. As in Lemma 5.1 of [S] (p.36), for any countable $\mathcal{R}$-module $\mathcal{M}$ there is a corresponding $\mathbf{Z}^{d}$-action $\alpha=\alpha^{\mathcal{M}}$ by automorphisms of the compact metric group $\widehat{\mathcal{M}}$. We may therefore write $n(\alpha)=n(\mathcal{M})$. By Theorem 27.2(1) of [S] (p.264) the mixing properties of $\alpha$ are determined by the mixing properties of the actions $\alpha^{\mathcal{R} / \mathcal{P}}$ corresponding to the prime ideals $\mathcal{P}$ of $\mathcal{R}$ associated with M. In particular

$$
n(\mathcal{M})=\min _{\mathcal{P}} n(\mathcal{R} / \mathcal{P})
$$

So in some sense it suffices to consider just these $\alpha=\alpha^{\mathcal{R} / \mathcal{P}}$. Certainly if $\mathcal{M}$ is Noetherian there are only finitely many $\mathcal{P}$ to consider, and it is well-known that these can often be effectively found (for example if $\mathcal{M}$ is an ideal of $\mathcal{R}$ ).

Then for $\alpha=\alpha^{\mathcal{R} / \mathcal{P}}$ a set $\left\{\mathbf{m}_{0}, \ldots, \mathbf{m}_{n}\right\}$ in $\mathbf{Z}^{d}$ of cardinality $n+1$ is non-mixing if and only if there are $a_{0}, \ldots, a_{n}$ in the quotient field $K$ of the integral domain $\mathcal{R} / \mathcal{P}$, not all zero, such that

$$
a_{0} \mathbf{u}^{\mathbf{m}_{0} k}+\cdots+a_{n} \mathbf{u}^{\mathbf{m}_{n} k}=0
$$

(in $K$ ) for infinitely many positive integers $k$, where $\mathbf{u}^{\mathbf{m}}=u_{1}^{m_{1}} \cdots u_{d}^{m_{d}}$ for $\mathbf{m}=\left(m_{1}, \ldots, m_{d}\right)$.

If the characteristic of $\mathcal{R} / \mathcal{P}$ is zero (so that $\mathcal{P} \cap \mathbf{Z}$ is zero) and $\alpha$ is mixing, then it is known that $\alpha$ is $n$-mixing for every $n \geq 3$. See Theorem 27.3(2) of [S] (p.265) for the proof, due to Schmidt and Ward [SW], which amounts to showing that is equivalent to the classical results of Evertse, Schlickewei and van der Poorten about linear equations over 
multiplicative groups in zero characteristic. Thus in this case $n(\mathcal{R} / \mathcal{P})$ must be either 1 or $\infty$.

The dichotomy here can be resolved in several ways; here is one possibility.

It is known that $\alpha^{\mathcal{R} / \mathcal{P}}$ is 2 -mixing (that is, just mixing) if and only if $u_{1}, \ldots, u_{d}$ stay multiplicatively independent in $K$. See for example Theorem 6.5(2) of [S] (p.47). Now it is not difficult to determine whether $u_{1}, \ldots, u_{d}$ become multiplicatively dependent modulo constants of $K$; a good estimate in terms of the variety in $\mathbf{C}^{d}$ associated with $\mathcal{P}$ is given as Lemma 3.2 of [BMZ] (p.14), for example. If there is such a dependence, then using a simple induction we can even determine all relations

$$
\mathbf{u}^{\mathbf{b}}=\beta
$$

in the form of a basis $\left(\mathbf{b}_{1}, \ldots, \mathbf{b}_{r}\right)$ for the group $B$ of all $\mathbf{b}=\left(b_{1}, \ldots, b_{d}\right)$ in $\mathbf{Z}^{d}$ for which there exists constant $\beta$ in (1.2). These $\beta$ must be algebraic over $\mathbf{Q}$; call them $\beta_{1}, \ldots, \beta_{r}$ corresponding to the basis elements. Now it is clear that $u_{1}, \ldots, u_{d}$ become multiplicatively dependent in $K$ if and only if $\beta_{1}, \ldots, \beta_{r}$ are themselves multiplicatively dependent. This latter can be determined in a standard way using heights; for a good estimate in a typical situation see Corollary 3.2 of [LM] (p.281) for example.

Let us assume that $\beta_{1}, \ldots, \beta_{r}$ are indeed multiplicatively dependent, so that the smallest order of non-mixing is 2 .

Then in a similar way one can determine the group of all $\mathbf{c}=\left(c_{1}, \ldots, c_{r}\right)$ in $\mathbf{Z}^{r}$ such that $\beta_{1}^{c_{1}} \cdots \beta_{r}^{c_{r}}=1$. Via $\mathbf{b}=c_{1} \mathbf{b}_{1}+\cdots+c_{r} \mathbf{b}_{r}$ this leads easily to the $\operatorname{subgroup} B_{1}$ of $B$ for which $\mathbf{u}^{\mathbf{b}}=1$. And $B_{1}$ is of finite index in a unique primitive subgroup $\sqrt{B_{1}}$ in $\mathbf{Z}^{d}$; this is the set of $\mathbf{b}$ for which there exists a root of unity $\zeta$ with

$$
\mathbf{u}^{\mathbf{b}}=\zeta
$$

Now it is an easy exercise using (1.1) to show that the set $\left\{\mathbf{m}_{0}, \mathbf{m}_{1}\right\}$ of cardinality 2 in $\mathbf{Z}^{d}$ is non-mixing for $\alpha$ if and only if the non-zero $\mathbf{m}_{0}-\mathbf{m}_{1}$ lies in $\sqrt{B_{1}}$.

Thus the only real problems arise when the characteristic $p$ of $R / \mathcal{P}$ is positive, and from now on we assume that this is the case. Then it is known that $n=n(\mathcal{R} / \mathcal{P})<\infty$ (see below).

When $n=1$ we can reason as in zero characteristic. Namely the arguments of Lemma 3.2 of [BMZ] stay valid in positive characteristic; the essential fact is that a field of rational 
functions over $\mathbf{F}_{p}$ in several variables is still a "field with a proper set of absolute values satisfying a product formula". Indeed this fact was used throughout [DM1] to define all the heights there. But then $\beta$ in (1.2) is algebraic over $\mathbf{F}_{p}$ and so a root of unity; thus we are automatically in (1.3).

Now by Theorem 28.7 (p.275) of [S] the non-mixing property of a set is invariant under $\mathbf{Z}^{d}$-translation and also under multiplication by a positive integer. Also from (1.1) it is trivially invariant under dividing by a positive integer as long as the set stays in $\mathbf{Z}^{d}$. Thus in particular it seems reasonable to think of the non-mixing sets as being in $\mathbf{Q}^{d}$ rather than $\mathbf{Z}^{d}$; further they fall into natural equivalence classes as follows.

Define two finite sets $M, M^{\#}$ in $\mathbf{Q}^{d}$ to be equivalent if there is a positive rational $x$, and $\mathbf{f}$ in $\mathbf{Q}^{d}$, such that $x M=M^{\#}+\mathbf{f}$ (this is not quite the same definition as in Ward [W] p.2). We might without much confusion describe the equivalence classes also as "shapes". Clearly every non-empty equivalence class contains an $M$ in $\mathbf{Z}^{d}$. We can even take all the coordinates non-negative, and moreover make sure that the convex hull touches every coordinate hyperplane (for example when $d=2$ we just push the set as far as it will go south and west). This is the same as saying that the Laurent polynomial $\sum_{\mathbf{m} \in M} u^{\mathbf{m}}$ is a genuine polynomial and not divisible by any of $u_{1}, \ldots, u_{d}$. We could call such a set semi-reduced.

We can further assume that no $s^{-1} M(s=2,3, \ldots)$ is in $\mathbf{Z}^{d}$; and this we call reduced.

It is not difficult to see that the reduced set in each class is unique (we will not need this until the examples). At first it is rather clear that if $M, M^{\#}$ are both semireduced and translates of each other, then they are equal (when $d=2$ this is obvious from pushing). And if $M, M^{\#}$ are both reduced and equivalent then $x M, M^{\#}$ are semi-reduced and translates so $x M=M^{\#}$. Writing $x=r / s$ for positive coprime $r, s$ we see that $\frac{r}{s} M$ is in $\mathbf{Z}^{d}$; but as $\frac{s}{s} M$ is too, so is $\frac{1}{s} M$ in $\mathbf{Z}^{d}$. Thus $s=1$. Similarly $r=1$ so $M=M^{\#}$ proving the uniqueness.

Here is the main result of this paper.

Theorem. Given a prime ideal $\mathcal{P}$ of $\mathcal{R}$ with $\mathcal{P} \cap \mathbf{Z}=p \mathbf{Z}(p>0)$ the smallest order $n+1=n(\mathcal{R} / \mathcal{P})+1<\infty$ of non-mixing can be effectively determined. Further if $n \geq 2$ then there are only finitely many equivalence classes of non- $(n+1)$-mixing sets, and these can be effectively determined.

The discussion above shows that the condition $n \geq 2$ is important for the finiteness. In fact the arguments above make it clear that a non-mixing action in positive characteristic 
can have infinitely many equivalence classes of non-mixing sets, but that this happens if and only if the rank $r$ of the analogue of the group $\sqrt{B_{1}}$ satisfies $r \geq 2$. For example this happens when $\mathcal{P}$ in $\mathcal{R}_{2}$ contains both $u_{1}-1$ and $u_{2}-1$, but not when $\mathcal{P}$ in $\mathcal{R}_{1}$ contains $u_{1}-1$.

We note that there is usually no trouble to find an effective upper bound for the smallest order of non-mixing, for example if $\mathcal{P}$ is explicitly given in terms of generators. Just pick any $P$ in $\mathcal{P}$ not in $p \mathcal{R}$, so that $P\left(u_{1}, \ldots, u_{d}\right)=0$ in $K$, and take the $k=p^{e}$ powers $(e=0,1,2, \ldots)$; the resulting equations then show by (1.1) that $\alpha$ is not $(N+1)$-mixing, where $N+1 \geq 2$ is the number of non-zero terms in $P$ reduced modulo $p$. So $n(\mathcal{R} / \mathcal{P}) \leq N$.

Thus it would seem that our work has something to do with the problem of finding the "shortest" polynomial in a given ideal; see also [S] p.282. In zero characteristic this problem is surprisingly difficult and probably there is in general no effective algorithm. In one variable it is related to a conjecture of Posner and Rumsey; see for example the article [SV] of Schlickewei and Viola, which makes use of the Subspace Theorem in the form of an $S$-unit equation. However the latter was one of the key objects in [D],[M] and [DM1], and the lesson there is that things are much easier in positive characteristic. In this case it is quite likely that the work in [DM1] leads to an effective solution of the shortest polynomial problem, although we do not investigate this in the present paper. But actually there is an extra twist here, which arises from the main result of $[\mathrm{M}]$. Namely we may have to extend the Laurent ring to a Puiseux ring.

A nice example of this is given in $[\mathrm{S}]$ (p.278). Here $\mathcal{P}$ is generated by $p=2$ and

$$
P=1+u_{1}+u_{1}^{3}+u_{1}^{5}+u_{1}^{6}+u_{2}=\left(1+u_{1}+u_{1}^{2}\right)^{3}+u_{2},
$$

where the shortest polynomial is probably $P$; at any rate $\alpha$ is not 6 -mixing. But $P$, although irreducible in $\mathbf{F}_{2}\left[u_{1}, u_{1}^{-1}, u_{2}, u_{2}^{-1}\right]$, is clearly divisible by

$$
Q=1+u_{1}+u_{1}^{2}+u_{2}^{\frac{1}{3}}
$$

in $\mathbf{F}_{2}\left[u_{1}, u_{2}^{\frac{1}{3}}\right]$. Now the $Q^{p^{e}}$ show equally well that $\alpha$ is not 4 -mixing (see section 4 ). The general situation for principal ideals $\mathcal{P}$ (when considered $\bmod p$ ) is clarified in terms of non-mixing sets by Proposition 28.9 of [S] (p.276). This shows how to find all non-mixing sets that are minimal in a certain sense. But it does not show how to find the ones of smallest cardinality. Here we illustrate our techniques by proving that $\alpha$ is 3 -mixing with exactly five classes of non-mixing sets of cardinality 4 . 
The other examples in [S] all concern principal ideals. Here we consider also a nonprincipal ideal. It is generated by 2 and

$$
P_{1}=1+u_{1}+u_{1}^{2}+u_{2}, \quad P_{2}=1+u_{1}+u_{1}^{3}+u_{3}
$$

Again there is certainly no 4-mixing, and again we will prove that there is 3 -mixing. But this time there are exactly 134 classes of non-mixing sets of cardinality 4 . The most complicated one comes from the fact that our ideal happens to contain

$$
u_{1}^{25}+u_{1}^{20} u_{2} u_{3}+u_{2}^{12}+u_{3}^{4} .
$$

These examples should make it clear that the determination of the smallest order of non-mixing and the equivalence classes of corresponding non-mixing sets is not only effective but also fairly practical. By using the estimates in [DM1] it should also be possible to give explicit bounds for the sets in terms of $\mathcal{P}$ or more precisely its generators.

Our proof uses observations from $[\mathrm{M}]$ as well as one of the main results of [DM1].

More precisely let $V$ be a variety in projective $n$-space defined by linear equations in $X_{0}, \ldots, X_{n}$ over positive characteristic. The work of [DM1] shows how to find all points of $V$ whose coordinates are in a given finitely generated group. The precise description can be complicated, involving (as we mentioned) as many as $n-1$ independently operating Frobenius maps, as well as cosets defined by equations $X_{i}=a X_{j}$ (see for example Theorem 1 of [DM1] p.1049).

But for an action $\alpha=\alpha^{\mathcal{R} / \mathcal{P}}$ as above with $n=n(\alpha)$, the hyperplane $V_{n}$ defined by the single equation $X_{0}+\cdots+X_{n}=0$, and the group as the radical inside $K$ of the group generated by $u_{1}, \ldots, u_{d}$ in $K$, the description is much simpler. In particular only a single Frobenius turns up, and apart from cosets we see only points $\left(\xi_{0}^{p^{e}}, \ldots, \xi_{n}^{p^{e}}\right)(e=0,1, \ldots)$ for a finite set $\Pi$ of $\left(\xi_{0}, \ldots, \xi_{n}\right)$. This is proved in Lemma 5 . It also shows that $\Pi$ is closely related to the desired equivalence classes; for that we need the concept of "broad set" used in $[\mathrm{M}]$, which is crucial to control the coefficients $a_{0}, \ldots, a_{n}$ in (1.1).

Our paper is arranged as follows. In section 2 we prove four lemmas as preparation for the fifth. Our Theorem follows quickly in section 3. Then section 4 treats the example (1.4) and section 5 the much more difficult (1.5).

We wish warmly to thank Klaus Schmidt for his interest in our work on orders of non-mixing and his encouragement to go further with the non-mixing sets themselves. 
2. Preliminaries. Let $k$ be any field (even of zero characteristic) and let $\mathcal{L}$ be a vector space of linear forms $L$ in variables $X_{0}, \ldots, X_{n}$. We say that $L=\sum_{i \in I} c_{i} X_{i}$ in $\mathcal{L}$ is minimal if there is no non-empty subset $I^{\prime} \neq I$ of $I$ such that some non-zero $L^{\prime}=\sum_{i \in I^{\prime}} c_{i}^{\prime} X_{i}$ is in $\mathcal{L}$.

Lemma 1. The space $\mathcal{L}$ is generated by its minimal forms.

Proof. Compare Lemma 4 of [BM] (p.431). It suffices to prove that every non-zero form $L$ in $\mathcal{L}$ can be written as a linear combination of minimal forms. This will be by induction on the length $l$ of $L$, that is, the number of non-zero coefficients. The case $l=1$ is trivial. So assume for some $l \geq 2$ that this holds for all forms of $\mathcal{L}$ of length strictly less than $l$. Take $L$ in $\mathcal{L}$ of length exactly $l$. After a permutation we can suppose $L=c_{0} X_{0}+\cdots+c_{l-1} X_{l-1}$. If $L$ is already minimal we are done. Otherwise we can assume after another permutation that some $L^{\prime}=c_{0}^{\prime} X_{0}+\cdots+c_{m-1}^{\prime} X_{m-1}$ lies in $\mathcal{L}$ with some $m<l$ and $c_{0}^{\prime} \neq 0$. Then $L^{\prime}$ and $L^{\prime \prime}=c_{0}^{\prime} L-c_{0} L^{\prime} \neq 0$ are both of length strictly less than $l$, and so the induction hypothesis can be applied to $L=\left(c_{0} / c_{0}^{\prime}\right) L^{\prime}+\left(1 / c_{0}^{\prime}\right) L^{\prime \prime}$ giving the required assertion for $L$. This proves the lemma.

With $\mathcal{R}$ and $\mathcal{P}$ as the Theorem, we work in the quotient field $K$ of $\mathcal{R} / \mathcal{P}$; then $\mathbf{F}=$ $\overline{\mathbf{F}_{p}} \cap K$ is a finite field. We also work with the group $G$ generated in $K^{*}$ by the images of $u_{1} \neq 0, \ldots, u_{d} \neq 0$. We write $\sqrt{G}$ for the radical of $G$ inside $K$. This is well-known to be finitely generated (see for example $[\mathrm{M}]$ p. 195). It clearly also has rank $d$; let $v_{1}, \ldots, v_{d}$ be basis elements modulo torsion (that is, modulo $\left.\mathbf{F}^{*}\right)$. For $\mathbf{m}=\left(m_{1}, \ldots, m_{d}\right)$ in $\mathbf{Z}^{d}$ we abbreviate $v_{1}^{m_{1}} \cdots v_{d}^{m_{d}}$ as above to $\mathbf{v}^{\mathbf{m}}$.

We write $\mathbf{P}_{n}(\sqrt{G})$ for the points of projective $n$-space $\mathbf{P}_{n}$ whose coordinates can be taken in $\sqrt{G}$. For a variety $V$ in $\mathbf{P}_{n}$ defined by linear equations we write $V(\sqrt{G})$ for the intersection $V \cap \mathbf{P}_{n}(\sqrt{G})$. We are going to use some results of [DMI], in which we say that $\psi$ from $\mathbf{P}_{n}$ to $\mathbf{P}_{n}$ is a $\sqrt{G}$-isomorphism if it is defined by $\psi\left(X_{0}, \ldots, X_{n}\right)=\left(g_{0} X_{0}, \ldots, g_{n} X_{n}\right)$ for $g_{0}, \ldots, g_{n}$ in $\sqrt{G}$; and that $V$ is $\sqrt{G}$-isotrivial if there is such a $\psi$ with $\psi(V)$ defined over F. We say that a variety is tranversal if each one of the projective variables $X_{0}, \ldots, X_{n}$ occurs in the defining equations with non-zero coefficient. We say that a variety is a torsion coset if it is defined by equations of the form $X_{i}=\zeta X_{j}(i \neq j)$ with $\zeta$ in $\mathbf{F}^{*}$. A transversal torsion coset $Z$ leads to a partition $I_{1} \cup \cdots \cup I_{h}$ of $\{0,1, \ldots, n\}$ into parts of size at least two together with $\zeta_{0}, \ldots, \zeta_{n}$ in $\mathbf{F}^{*}$, such that for each $j=1, \ldots, h$ the equality of the quotients $X_{i} / \zeta_{i}\left(i \in I_{j}\right)$ defines $Z$. We define the variety $V_{n}$ by $X_{0}+\cdots+X_{n}=0$. 
Lemma 2. Suppose for some $n \geq 1$ that $\alpha$ is $(n+1)$-mixing. Then there exists a finite collection $\mathcal{Z}$ of transversal torsion cosets $Z$ in $V_{n}$ such that

$$
V_{n}(\sqrt{G})=\bigcup_{Z \in \mathcal{Z}} Z(\sqrt{G})
$$

Proof. This bears some resemblance to the Descent Step (a) over $\sqrt{G}$ of [DM1] (p.1047). However we cannot apply it here because $V_{n}$ is not only $\sqrt{G}$-isotrivial but even defined over $\mathbf{F}_{p}$. The proof that follows is self-contained. Take any point $\left(\xi_{0}, \ldots, \xi_{n}\right)$ of $V_{n}(\sqrt{G})$, and write $\xi_{i}=\zeta_{i} \mathbf{v}^{\mathbf{r}_{i}}$ for torsion $\zeta_{i}(i=0,1, \ldots, n)$. Frobenius leads to

$$
\zeta_{0} \mathbf{v}^{\mathbf{r}_{0} q}+\cdots+\zeta_{n} \mathbf{v}^{\mathbf{r}_{n} q}=0
$$

for infinitely many prime powers $q=p^{e}$. We convert this into powers $\mathbf{u}^{\mathbf{m}}$ by a standard argument. Let $s=[\sqrt{G}: G]$. There is some $r$ such that $q$ is congruent to $r$ modulo $s$ for infinitely many $q$, and we get

$$
a_{0} \mathbf{u}^{\mathbf{m}_{0} k}+\cdots+a_{n} \mathbf{u}^{\mathbf{m}_{n} k}=0 \quad(k=(q-r) / s)
$$

(in $K$ ) for these $q$, where

$$
a_{0}=\zeta_{0} \mathbf{v}^{\mathbf{r}_{0} r}, \ldots, a_{n}=\zeta_{n} \mathbf{v}^{\mathbf{r}_{n} r}
$$

and $\mathbf{m}_{0}, \ldots, \mathbf{m}_{n}$ are defined by

$$
\mathbf{v}^{\mathbf{r}_{0} s}=\mathbf{u}^{\mathbf{m}_{0}}, \ldots, \mathbf{v}^{\mathbf{r}_{n} s}=\mathbf{u}^{\mathbf{m}_{n}} .
$$

As $k \rightarrow \infty$ in (2.1) this looks suspiciously like non- $(n+1)$-mixing (1.1), even with a non- $(n+1)$-mixing set $M=\left\{\mathbf{m}_{0}, \ldots, \mathbf{m}_{n}\right\}$. The only way out is that $M$ has cardinality $h<n+1$. Writing $M=\left\{\mathbf{m}_{1}^{\prime}, \ldots, \mathbf{m}_{h}^{\prime}\right\}$ and $I_{j}$ for the set of $i$ with $\mathbf{m}_{i}=\mathbf{m}_{j}^{\prime}$ we get a partition $I_{1} \cup \cdots \cup I_{h}$ of $\{0,1, \ldots, n\}$. Then

$$
0=\sum_{i=0}^{n} a_{i} \mathbf{u}^{\mathbf{m}_{i} k}=\sum_{j=1}^{h} b_{j} \mathbf{u}_{j}^{\mathbf{m}_{j}^{\prime} k}
$$

with $b_{j}=\sum_{i \in I_{j}} a_{i}$. Even then this looks like non- $h$-mixing; but this time the only way out is $b_{1}=\cdots=b_{h}=0$.

In particular each $I_{j}$ has cardinality at least two, and the quantities $\mathbf{v}^{\mathbf{r}_{i} s}=\mathbf{u}^{\mathbf{m}_{i}}=$ $\mathbf{u}^{\mathbf{m}_{j}^{\prime}}\left(i \in I_{j}\right)$ are equal, so also the $\mathbf{r}_{i}\left(i \in I_{j}\right)$, say to $\mathbf{m}_{j}^{\prime \prime}$. So also the

$$
\frac{\xi_{i}}{\zeta_{i}}=\mathbf{v}^{\mathbf{r}_{i}}=\mathbf{v}^{\mathbf{m}_{j}^{\prime \prime}} \quad\left(i \in I_{j}\right)
$$


Thus our point $\left(\xi_{0}, \ldots, \xi_{n}\right)$ lies in the corresponding transversal torsion coset $Z$. Also

$$
\sum_{i \in I_{j}} \zeta_{i}=\sum_{i \in I_{j}} a_{i} \mathbf{v}^{-\mathbf{r}_{i} r}=\mathbf{v}^{-\mathbf{m}_{j}^{\prime \prime} r} \sum_{i \in I_{j}} a_{i}=0 \quad(j=1, \ldots, h)
$$

and this implies that $Z$ lies in $V_{n}$. That completes the proof.

If $\alpha$ is only $n$-mixing, then we cannot expect a conclusion as strong as that of Lemma 2. But the following is not too much weaker, where for $\pi=\left(\xi_{0}, \ldots, \xi_{n}\right)$ we write $\pi^{l}=$ $\left(\xi_{0}^{l}, \ldots, \xi_{n}^{l}\right)$.

Lemma 3. Suppose for some $n \geq 2$ that $\alpha$ is $n$-mixing. Then there exists a finite collection $\mathcal{Z}$ of transversal torsion cosets $Z$ in $V_{n}$ and a finite set $\Pi$ in $\mathbf{P}_{n}(\sqrt{G})$ such that

$$
V_{n}(\sqrt{G})=\bigcup_{Z \in \mathcal{Z}} Z(\sqrt{G}) \cup \bigcup_{\pi \in \Pi} \bigcup_{e=0}^{\infty} \pi^{p^{e}}
$$

Proof. We apply the Descent Step (b) over $\sqrt{G}$ of [DM1] (p.1047) with $\psi$ there as the identity and $q$ there as $p$. Because $V_{n}$ is not a coset, we obtain a finite collection $\mathcal{W}$ of proper $\sqrt{G}$-isotrivial linear subvarieties $W \neq V_{n}$ of $V_{n}$, also defined over $K$, such that

$$
V_{n}(\sqrt{G})=\bigcup_{W \in \mathcal{W}} \bigcup_{e=0}^{\infty} W(\sqrt{G})^{p^{e}} .
$$

It will turn out that all the $W$ here which are positive-dimensional can be taken as transversal torsion cosets.

Consider any $W$ in (2.2), and pick a $\sqrt{G}$-isomorphism $\psi$ with $\psi(W)$ defined over $\mathbf{F}$. We call $W$ minimal if

$$
W(\sqrt{G}) \neq \bigcup_{W^{\prime} \in \mathcal{W}^{\prime}} W^{\prime}(\sqrt{G})
$$

for a finite collection $\mathcal{W}^{\prime}$ of $\sqrt{G}$-isotrivial linear subvarieties $W^{\prime} \neq W$ of $W$ with $\psi\left(W^{\prime}\right)$ defined over $\mathbf{F}$. If some $W$ is not minimal then we can replace it in (2.2) by lowerdimensional varieties. So we can assume here that all $W$ are minimal and of course that all $W(\sqrt{G})$ are non-empty.

Consider such a $W$ with $\tilde{W}=\psi(W)$ defined over $\mathbf{F}$. Say

$$
\psi\left(X_{0}, \ldots, X_{n}\right)=\left(g_{0} X_{0}, \ldots, g_{n} X_{n}\right)=\left(\tilde{X}_{0}, \ldots, \tilde{X}_{n}\right)
$$


for $g_{0}, \ldots, g_{n}$ in $\sqrt{G}$. We know from Lemma 1 that the ideal of $\tilde{W}$ is generated by the minimal forms. Let $\sum_{i \in I} \zeta_{i} \tilde{X}_{i}$ be one of these, with of course $\zeta_{i} \neq 0$ in $\mathbf{F}$. As $\operatorname{dim} \tilde{W}<$ $\operatorname{dim} V_{n}=n-1$, any $n$ from $\tilde{X}_{0}, \ldots, \tilde{X}_{n}$ are dependent over $\mathbf{F}$ on $\tilde{W}$, and so $I$ has cardinality at most $n$. Pick any $\left(\xi_{0}, \ldots, \xi_{n}\right)$ in $W(\sqrt{G})$. We get $\sum_{i \in I} \zeta_{i} g_{i} \xi_{i}=0$. So by Lemma 2 (in lower dimension) some $\left(\zeta_{i} g_{i} \xi_{i}\right) /\left(\zeta_{j} g_{j} \xi_{j}\right)(i, j \in I, i \neq j)$ lies in $\mathbf{F}^{*}$, say $\zeta$. It follows that

$$
W(\sqrt{G})=\bigcup_{i j \zeta} W_{i j \zeta}(\sqrt{G}),
$$

where $W_{i j \zeta}$ is defined by $\zeta_{i} g_{i} X_{i}=\zeta \zeta_{j} g_{j} X_{j}$ in $W$. If all $W_{i j \zeta}$ here are $\neq W$, we contradict the minimality of $W$. So some $W_{i j \zeta}=W$. This means that $\zeta_{i} \tilde{X}_{i}-\zeta \zeta_{j} \tilde{X}_{j}$ vanishes on $\tilde{W}$. Thus that must have been the minimal form from the start.

Therefore $\tilde{W}$ is a torsion coset. As it lies in the transversal $\psi\left(V_{n}\right)$ it must be itself transversal. Thus it comes from a partition $I_{1} \cup \cdots \cup I_{h}$ of $\{0,1, \ldots, n\}$ into parts of size at least two together with $\tilde{\zeta}_{i}$ in $\mathbf{F}^{*}$, such that for each $j=1, \ldots, h$ the equality of the quotients $\tilde{X}_{i} / \tilde{\zeta}_{i}\left(i \in I_{j}\right)$ defines $\tilde{W}$. So $W$ is defined by the corresponding equality of the $X_{i} / \tilde{g}_{i}\left(i \in I_{j}\right)$, where the $\tilde{g}_{i}=\tilde{\zeta}_{i} / g_{i}$ are still in $\sqrt{G}$.

Now the fact that $W$ lies in $V_{n}$ is easily seen to imply the equations

$$
\sum_{i \in I_{j}} \tilde{g}_{i}=0 \quad(j=1, \ldots, h) .
$$

If $h=1$ then of course $W$ is the point $\pi=\left(\tilde{g}_{0}, \ldots, \tilde{g}_{n}\right)$, and we define $\Pi$ as the finite set of points arising in this way.

So we assume $h \geq 2$ from now on. Now every sum in (2.3) involves at most $n$ terms, so Lemma 2 is applicable as above. It yields a further partition $I_{j}=\bigcup I_{j k}$ into parts of size at least two together with more $\zeta_{i}^{\prime}$ in $\mathbf{F}^{*}$ such that the $\tilde{g}_{i} / \zeta_{i}^{\prime}\left(i \in I_{j k}\right)$ are equal. Further just as in (2.3) we get $\sum_{i \in I_{j k}} \zeta_{i}^{\prime}=0$.

Consider now the linear variety $Z_{W}$ defined by the equality of the $X_{i} / \zeta_{i}^{\prime}\left(i \in I_{j k}\right)$ for each choice of $j, k$. It is a transversal torsion coset contained in $V_{n}$. It is not difficult to check that $W$ lies in $Z_{W}$ (but there might be more equations defining $W$, for example those connecting $X_{i}$ in $I_{j k}$ for different $k$ ). At any rate we have for each $\pi$ in $\Pi$

$$
W(\sqrt{G}) \cup\{\pi\} \subseteq Z_{W}(\sqrt{G}) \cup\{\pi\} \subseteq V_{n}(\sqrt{G}) .
$$

Raising to the power $p^{e}$ and taking the union over all positive-dimensional $W$ in $\mathcal{W}$, all $\pi$ in $\Pi$, and $e=0,1,2, \ldots$, we get $V_{n}(\sqrt{G})$ not only on the right but also on the left, by 
(2.2). It follows that this is also the middle term

$$
\bigcup_{W} \bigcup_{e=0}^{\infty} Z_{W}(\sqrt{G})^{p^{e}} \cup \bigcup_{\pi \in \Pi} \bigcup_{e=0}^{\infty} \pi^{p^{e}} .
$$

Now this completes the proof since each $Z_{W}$ has only a finite set $\mathcal{Z}_{W}$ of conjugates over $\mathbf{F}_{p}$, and

$$
\bigcup_{e=0}^{\infty} Z_{W}(\sqrt{G})^{p^{e}}=\bigcup_{Z \in \mathcal{Z}_{w}} Z(\sqrt{G}) .
$$

The following observation is crucial to get information about the coefficients arising from non-mixing. It seems convenient to work affinely for a bit. Recall from $[\mathrm{M}]$ (p.189) that a set $\Sigma$ in $\sqrt{G}^{n}$ is called broad if

(i) $\Sigma$ is infinite,

(ii) for each $g$ in $\sqrt{G}$ and each $i=1, \ldots, n$ there are at most finitely many $\left(x_{1}, \ldots, x_{n}\right)$ in $\Sigma$ with $x_{i}=g$,

(iii) if $n \geq 2$ then for each $g$ in $\sqrt{G}$ and each $i, j=1, \ldots, n$ with $i \neq j$ there are at most finitely many $\left(x_{1}, \ldots, x_{n}\right)$ in $\Sigma$ with $x_{i} / x_{j}=g$.

Lemma 4. Suppose for some $n \geq 2$ that $\alpha$ is $n$-mixing, and that there exist $a_{1}, \ldots, a_{n}$ in $K$ such that the equation $a_{1} x_{1}+\cdots+a_{n} x_{n}=1$ has a broad set of solutions in $(\sqrt{G})^{n}$. Then $a_{1}, \ldots, a_{n}$ lie in $\sqrt{G}$.

Proof. The $a_{1}, \ldots, a_{n}$ lie in $K^{*}$ otherwise we would have non- $n$-mixing. This can be seen by writing the solutions as $x_{i}=\zeta_{i} \mathbf{v}^{\mathbf{m}_{i}}(i=1, \ldots, n)$ for $\zeta_{i}$ in $\mathbf{F}^{*}$ and then getting into $G$ by reducing the exponents modulo $s$ according to $\mathbf{m}_{i}=\mathbf{m}_{i 0}+s \mathbf{q}_{i}$; then $\mathbf{v}^{s \mathbf{q}_{i}}=\mathbf{u}^{\mathbf{p}_{i}}$. If say $a_{n}=0$ we get equations $a_{1}^{\prime} \mathbf{u}^{\mathbf{p}_{1}}+\cdots+a_{n-1}^{\prime} \mathbf{u}^{\mathbf{p}_{n-1}}=1$ (in $K$ ) and now these give $n$-mixing (see [S] p.263) unless some $\mathbf{p}_{i}$ or some $\mathbf{p}_{i}-\mathbf{p}_{j}(i \neq j)$ does not tend to infinity. But this would contradict the broadness.

Therefore $a_{1}, \ldots, a_{n}$ satisfy the hypotheses of Lemma 5 of $[\mathrm{M}]$ (p.197) with $\sqrt{G}$ in place of $G$.

Thus either (aa) or (bb) of Lemma 5 holds. But (aa) would also lead to non- $n$-mixing. So (bb) holds.

Now apply Lemma 5 to the new equation (16) of [M] (p.198). Again (bb) must hold.

And so on for ever. By Lemma 2 of $[\mathrm{M}]$ (p.193) this means that $a_{1}, \ldots, a_{n}$ must lie in $\sqrt{G}$. This proves the present lemma. 
We call the projective $\left(\xi_{0}, \ldots, \xi_{n}\right)$ with non-zero coordinates pre-broad if no $\xi_{i} / \xi_{j}(i \neq$ $j$ ) lies in $\mathbf{F}^{*}$. We call two such points $\pi, \pi^{\#}$ proportional if there are positive integers $l, l^{\#}$ with $\pi^{l^{\#}}=\pi^{\# l}$.

We note that the equation defining $V_{n}$ is invariant under the symmetric group $S_{n+1}$ on $n+1$ elements, so that this also acts on points of $V_{n}$. It also acts on proportionality classes.

Lemma 5. Suppose for some $n \geq 2$ that $\alpha$ is n-mixing but not $(n+1)$-mixing. Then there exists a finite collection $\mathcal{Z}$ of transversal torsion cosets $Z$ in $V_{n}$, and a finite set $\Pi$ in $\mathbf{P}_{n}(\sqrt{G})$, containing at least one pre-broad element, such that

$$
V_{n}(\sqrt{G})=\bigcup_{Z \in \mathcal{Z}} Z(\sqrt{G}) \cup \bigcup_{\pi \in \Pi} \bigcup_{e=0}^{\infty} \pi^{p^{e}}
$$

Further $\alpha$ has only finitely many equivalence classes of non- $(n+1)$-mixing sets, and these are in one-to-one correspondence with the $S_{n+1}$-orbits of the proportionality classes of the pre-broad $\pi$ in $\Pi$.

Proof. Because $\alpha$ is non- $(n+1)$-mixing, there certainly exist non- $(n+1)$-mixing sets. Pick any such set. It is equivalent to some $\left\{0, \mathbf{m}_{1}, \ldots, \mathbf{m}_{n}\right\}$ in $\mathbf{Z}^{d}$ and then there are $a_{1}, \ldots, a_{n}$ in $K$ and an infinite set of positive integers $k$ such that

$$
a_{1} \mathbf{u}^{\mathbf{m}_{1} k}+\cdots+a_{n} \mathbf{u}^{\mathbf{m}_{n} k}=1
$$

(in $K$ ). As there is 2-mixing, the $u_{1}, \ldots, u_{d}$ are multiplicatively independent in $K$, and in particular the hypotheses of Lemma 4 are satisfied. It follows that $a_{1}, \ldots, a_{n}$ lie in $\sqrt{G}$.

With the basis elements $v_{1}, \ldots, v_{d}$ of $\sqrt{G}$ modulo torsion we can write $a_{i}=\zeta_{i} \mathbf{v}^{\mathbf{p}_{i}}(i=$ $1, \ldots, n)$ and

$$
u_{h}=\zeta_{h}^{\prime} \mathbf{v}^{\mathbf{q}_{h}} \quad(h=1, \ldots, d)
$$

for $\zeta_{i}, \zeta_{l}^{\prime}$ in $\mathbf{F}^{*}$. Putting the rows $\mathbf{q}_{1}, \ldots, \mathbf{q}_{d}$ together to make an invertible integral matrix $\mathbf{Q}$, so that $\mathbf{u}^{\mathbf{m}}=\zeta_{\mathbf{m}} \mathbf{v}^{\mathbf{m Q}}$ for $\zeta_{\mathbf{m}}$ in $\mathbf{F}^{*}$, we obtain the points

$$
\left(\xi_{0}^{(k)}, \xi_{1}^{(k)}, \ldots, \xi_{n}^{(k)}\right)=\pi^{(k)}=\left(-1, \zeta_{1}^{(k)} \mathbf{v}^{\mathbf{p}_{1}+\mathbf{m}_{1} \mathbf{Q} k}, \ldots, \zeta_{n}^{(k)} \mathbf{v}^{\mathbf{p}_{n}+\mathbf{m}_{n} \mathbf{Q} k}\right)
$$

on $V_{n}$ as in Lemma 3, with $\zeta_{i}^{(k)}$ in $\mathbf{F}^{*}$. We are going to prove that there cannot exist two different $k, k^{\prime}$ such that $\pi^{(k)}, \pi^{\left(k^{\prime}\right)}$ lie in the same $Z$. 
If $\pi^{(k)}$ lies in $Z$ then some $\xi_{i}^{(k)} / \xi_{j}^{(k)}(i \neq j)$ would be in $\mathbf{F}^{*}$. If for example $i \neq 0, j \neq 0$ then this implies that $\left(\zeta_{i}^{(k)} \mathbf{v}^{\mathbf{p}_{i}+\mathbf{m}_{i} \mathbf{Q} k}\right) /\left(\zeta_{j}^{(k)} \mathbf{v}^{\mathbf{p}_{j}+\mathbf{m}_{j} \mathbf{Q} k}\right)$ lies in $\mathbf{F}^{*}$ and so $\mathbf{p}_{i}+\mathbf{m}_{i} \mathbf{Q} k=$ $\mathbf{p}_{j}+\mathbf{m}_{j} \mathbf{Q} k$, that is, $\mathbf{p}_{i}-\mathbf{p}_{j}=-\left(\mathbf{m}_{i}-\mathbf{m}_{j}\right) \mathbf{Q} k$. Writing the same equation for $k^{\prime} \neq k$ and subtracting gives a contradiction as $\mathbf{m}_{i} \neq \mathbf{m}_{j}$. A similar argument works if $i=0$ or $j=0$ (with $\mathbf{m}_{0}=0$ ).

Thus for all sufficiently large $k$ the points $\pi^{(k)}$ must be the $\pi^{q}$ for $\pi$ in $\Pi$ and $q=p^{e}(e=$ $0,1,2, \ldots$,$) . So we can find two different k, k^{\prime}$ and two $q, q^{\prime}$ with $\pi^{(k)}=\pi^{q}, \pi^{\left(k^{\prime}\right)}=\pi^{q^{\prime}}$ for the same $\pi=\left(-1, \xi_{1}, \ldots, \xi_{n}\right)$ in $\Pi$. Writing $\xi_{i}=\tilde{\zeta}_{i} \mathbf{v}^{\mathbf{r}_{i}}$ for $\tilde{\zeta}_{i}$ in $\mathbf{F}^{*}$, we get as above

$$
\mathbf{p}_{i}+\mathbf{m}_{i} \mathbf{Q} k=\mathbf{r}_{i} q, \quad \mathbf{p}_{i}+\mathbf{m}_{i} \mathbf{Q} k^{\prime}=\mathbf{r}_{i} q^{\prime} \quad(i=1, \ldots, n) .
$$

Thus

$$
\mathbf{m}_{i} \mathbf{Q}\left(k-k^{\prime}\right)=\mathbf{r}_{i}\left(q-q^{\prime}\right) \quad(i=1, \ldots, n) .
$$

In particular $q \neq q^{\prime}$, and so our set $\left\{0, \mathbf{m}_{1}, \ldots, \mathbf{m}_{n}\right\}$ is equivalent to

$$
\pm\left\{0, \mathbf{r}_{1}, \ldots, \mathbf{r}_{n}\right\} \mathbf{Q}^{-1} \text {. }
$$

As $\mathbf{Q}$ is fixed and there are only finitely many possibilities for $\left\{0, \mathbf{r}_{1}, \ldots, \mathbf{r}_{n}\right\}$ corresponding to the finite set $\Pi$, the finiteness assertion in the present lemma for non-mixing sets follows.

The existence of some pre-broad $\pi$ in $\Pi$ also follows, because the point

$$
\pi=\left(-1, \xi_{1}, \ldots, \xi_{n}\right)=\left(-1, \tilde{\zeta}_{1} \mathbf{v}^{\mathbf{r}_{1}}, \ldots, \tilde{\zeta}_{n} \mathbf{v}^{\mathbf{r}_{n}}\right)
$$

is pre-broad if and only if the set $\left\{0, \mathbf{r}_{1}, \ldots, \mathbf{r}_{n}\right\}$ has cardinality $n+1$.

But to prove the one-to-one assertion we must tighten things up a bit.

We first show how to eliminate the minus possibility in (2.6). We can suppose that the $k, k^{\prime}$ above are just two elements of an infinite set. We fix $k$ and then make $k^{\prime}$ tend to infinity. Using heights as in [M],[DM1] we can easily see (from $\mathbf{m}_{n} \neq 0$ for example) that the height of $\pi^{\left(k^{\prime}\right)}$ tends to infinity. Thus the corresponding $q^{\prime}$ tends to infinity. Therefore we can assume $k<k^{\prime}$ and $q<q^{\prime}$. So indeed we can improve (2.6) to $\left\{0, \mathbf{r}_{1}, \ldots, \mathbf{r}_{n}\right\} \mathbf{Q}^{-1}$.

We described above how a non-mixing set $M$ gives rise to $\pi$. Suppose two such sets $M, M^{\#}$ give rise to $\pi, \pi^{\#}$ in the same $S_{n+1}$-orbit of proportionality classes. We show that $M, M^{\#}$ are equivalent.

Say $\pi^{\# l}=\left((-1)^{l}, \tilde{\zeta}_{1}^{\# l} \mathbf{v}_{1}^{\mathbf{r}_{1} l}, \ldots, \tilde{\zeta}_{n}^{\# l} \mathbf{v}_{n}^{\mathbf{r}_{n} l}\right)$ comes, for example, from permuting the first two coordinates of $\pi^{l^{\#}}=\left((-1)^{l^{\#}}, \tilde{\zeta}_{1}^{l^{\#}} \mathbf{v}^{\mathbf{r}_{1} l^{\#}}, \ldots, \tilde{\zeta}_{n}^{\#} \mathbf{v}^{\mathbf{r}_{n} l^{\#}}\right)$. Then

$$
\tilde{\zeta}_{1}^{\# l} \mathbf{v}_{1}^{\mathbf{r}_{1}^{\#} l}=(-1)^{l^{\#}-l} \tilde{\zeta}_{1}^{-l^{\#}} \mathbf{v}^{-\mathbf{r}_{1} l^{\#}}
$$




$$
\tilde{\zeta}_{i}^{\# l} \mathbf{v}^{\mathbf{r}_{i}^{\#} l}=(-1)^{-l} \tilde{\zeta}_{1}^{-l^{\#}} \mathbf{v}^{-\mathbf{r}_{1} l^{\#}} \tilde{\zeta}_{i}^{l^{\#}} \mathbf{v}^{\mathbf{r}_{i} l^{\#}} \quad(i=2, \ldots, n)
$$

In particular

$$
\begin{gathered}
\mathbf{r}_{1}^{\#} l=-\mathbf{r}_{1} l^{\#} \\
\mathbf{r}_{i}^{\#} l=-\mathbf{r}_{1} l^{\#}+\mathbf{r}_{i} l^{\#} \quad(i=2, \ldots, n) .
\end{gathered}
$$

Thus looking at the improved (2.6) we see that $M^{\#}$ is equivalent to

$$
\left\{0,-\mathbf{r}_{1},-\mathbf{r}_{1}+\mathbf{r}_{2}, \ldots,-\mathbf{r}_{1}+\mathbf{r}_{n}\right\} \mathbf{Q}^{-1}
$$

in turn equivalent to $\left\{\mathbf{r}_{1}, 0, \mathbf{r}_{2}, \ldots, \mathbf{r}_{n}\right\} \mathbf{Q}^{-1}$ and so to $M$.

A similar argument works for any permutation, and thus the number of classes of non-mixing sets is at most the number of orbits.

To prove the opposite inequality we note as in the proof of Lemma 2 that a pre-broad $\pi=\left(-1, \tilde{\zeta}_{1} \mathbf{v}^{\mathbf{r}_{1}}, \ldots, \tilde{\zeta}_{n} \mathbf{v}^{\mathbf{r}_{n}}\right)$ gives rise to a potential non-mixing set via

$$
\tilde{\zeta}_{1} \mathbf{v}^{\mathbf{r}_{1} q}+\cdots+\tilde{\zeta}_{n} \mathbf{v}^{\mathbf{r}_{n} q}=1
$$

and $k=(q-r) / s$ to get

$$
a_{1} \mathbf{u}^{\mathbf{m}_{1} k}+\cdots+a_{n} \mathbf{u}^{\mathbf{m}_{n} k}=1
$$

(in $K$ ) for

$$
a_{i}=\tilde{\zeta}_{i} \mathbf{v}^{\mathbf{r}_{i} r}, \quad \mathbf{u}^{\mathbf{m}_{i}}=\mathbf{v}^{\mathbf{r}_{i} s} \quad(i=1, \ldots, n) .
$$

Using (2.4) we see that $\mathbf{u}^{\mathbf{m}}=\zeta_{\mathbf{m}} \mathbf{v}^{\mathbf{m Q}}$ for some torsion $\zeta_{\mathbf{m}}$, and it follows that

$$
\mathbf{m}_{i} \mathbf{Q}=\mathbf{r}_{i} s \quad(i=1, \ldots, n)
$$

consistent with (2.5). As $\pi$ is pre-broad, the set $M=\left\{0, \mathbf{m}_{1}, \ldots, \mathbf{m}_{n}\right\}$ has cardinality $n+1$ and is therefore indeed non-mixing.

As above it is now rather easy to see that if $\pi, \pi^{\#}$ give rise to equivalent $M, M^{\#}$ then they are in the same orbit, so we get the desired opposite inequality. Here it is convenient to note that any $\pi$ is in the same orbit as some power $\pi^{l}=\left(1, \mathbf{v}^{\mathbf{s}_{1}}, \ldots, \mathbf{v}^{\mathbf{s}_{n}}\right)$ and so the roots of unity play no role.

3. Proof of Theorem. Because $n \geq 2$, our $\alpha=\alpha^{\mathcal{R} / \mathcal{P}}$ is 2-mixing. In the notation of the previous section, we look first at $V_{2}(\sqrt{G})$. From Lemma 3 we get $\mathcal{Z}$ and $\Pi$. 
If some $\pi=\left(\xi_{0}, \xi_{1}, \xi_{2}\right)$ in $\Pi$ is pre-broad, then $\alpha$ is non-3-mixing, else Lemma 2 would show that this $\pi$ lies in some transversal torsion coset, forcing some $\xi_{i} / \xi_{j}(i \neq j)$ in $\mathbf{F}^{*}$. So 3 is the smallest order of non-mixing, and by Lemma 5 there are only finitely many classes.

Otherwise no $\pi$ in $\Pi$ is pre-broad, and now Lemma 5 shows that $\alpha$ must be 3 -mixing. We then jump to $V_{3}(\sqrt{G})$ and repeat the process. Eventually we must find some pre-broad point in some $\Pi$ corresponding to some $V_{n}(\sqrt{G})$, and this leads to non- $(n+1)$-mixing. This $n+1$ is the required smallest order of non-mixing. And as explained in section 1 , an a priori upper bound can be found in the usual way simply by taking any non-zero polynomal in $\mathcal{P}$. This completes the proof of the Theorem.

The effectivity follows at once from the effectivity of [DM1].

4. An example. Before starting with this, we consider briefly the original Ledrappier example [Led], which is 2 -mixing but not 3 -mixing. It corresponds to $\mathcal{P}$ generated by 2 and $1+u_{1}+u_{2}$ in $\mathcal{R}=\mathbf{Z}\left[u_{1}, u_{1}^{-1}, u_{2}, u_{2}^{-1}\right]$. The group $G$ has generators the images of $u_{1}, u_{2}$ in the quotient field $K$ of $\mathcal{R} / \mathcal{P}$. We may identify $K$ with $\mathbf{F}_{2}(t)$ and the generators with $t, 1+t$ respectively. As these are clearly multiplicatively independent, we see already that $\alpha$ is 2-mixing. Equally clearly $\sqrt{G}$ has generators $t, 1+t$. To go further we need the field $C=\mathbf{F}_{2}\left(t^{2}\right)$ of differential constants.

Now Leitner in Theorem 1 (p.327) of [Lei] shows that

$$
V_{2}(\sqrt{G})=\bigcup_{\pi \in S_{3}\left(\pi_{0}\right)} \bigcup_{e=0}^{\infty} \pi^{2^{e}}
$$

for $\pi_{0}=(1, t, 1+t)$. Thus we see at once from Lemma 5 and $(2.7)$ that there is exactly one class of non-mixing sets of order 3 , with representative $\{(0,0),(1,0),(0,1)\}$. See also Lemma 5.6 (p.348) of the paper [ABB] of Arenas-Carmona, Berend and Bergelson (which is however more concerned with higher order mixing for Ledrappier away from this shape).

It might be fun to try $\mathcal{P}$ generated by 2 and $1+u_{1}+u_{2}+u_{3}$ in $\mathbf{Z}\left[u_{1}, u_{1}^{-1}, u_{2}, u_{2}^{-1}, u_{3}, u_{3}^{-1}\right]$. But perhaps one should glance at section 5 before starting. And one would have to work with two variables $t, t^{\prime}$.

We return to the example of $[\mathrm{S}]$ (p.278). Here $\mathcal{P}$ is generated by 2 and (1.4) in $\mathcal{R}=\mathbf{Z}\left[u_{1}, u_{1}^{-1}, u_{2}, u_{2}^{-1}\right]$. As already remarked, the factor $1+u_{1}+u_{1}^{2}+u_{2}^{\frac{1}{3}}$ in $\mathbf{F}_{2}\left[u_{1}, u_{2}^{\frac{1}{3}}\right]$ shows that $\alpha$ is not 4 -mixing, because in the quotient we have

$$
0=1+u_{1}^{2^{e}}+u_{1}^{2.2^{e}}+u_{2}^{2^{e} / 3}=1+a_{1} u_{1}^{3 k}+a_{2} u_{1}^{6 k}+a_{3} u_{2}^{k}
$$


for all even $e$, with $a_{1}=u_{1}, a_{2}=u_{1}^{2}, a_{3}=u_{2}^{\frac{1}{3}}=1+u_{1}+u_{1}^{2}$ in $K$ and $k=\left(2^{e}-1\right) / 3$; and so the non-mixing set

$$
\{(0,0),(3,0),(6,0),(0,1)\} .
$$

We will prove here that $\alpha$ is 3 -mixing and that there are exactly five equivalence classes for non-mixing sets of size 4 .

The group $G$ has generators the images of $u_{1}, u_{2}$ in the quotient field $K$ of $\mathcal{R} / \mathcal{P}$. We may again identify $K$ with $\mathbf{F}_{2}(t)$ and the generators with $t,\left(1+t+t^{2}\right)^{3}$ respectively. As these are clearly multiplicatively independent, we see already that $\alpha$ is 2 -mixing. It is easy to see that $\sqrt{G}$ has generators $t, 1+t+t^{2}$.

Already by Lemma 5 above with $n=2$, the next lemma shows that $\alpha$ is 3 -mixing.

Lemma 6. The set $V_{2}(\sqrt{G})$ is empty.

Proof. It suffices to deduce a contradiction from the existence of $x$ and $y$ in $\sqrt{G}$ with $x+y=1$. We follow the methods of [Lei].

Assume first that the $C$-vector space $C x+C y$ has dimension 2. Using a dot to indicate the derivative with respect to $t$, we deduce $\dot{y} / y \neq \dot{x} / x$. We get in the usual way the identities

$$
x=\frac{\frac{\dot{y}}{y}}{\frac{\dot{y}}{y}-\frac{\dot{x}}{x}}, \quad y=\frac{-\frac{\dot{x}}{x}}{\frac{\dot{y}}{y}-\frac{\dot{x}}{x}} .
$$

Now if $z=t^{a}\left(1+t+t^{2}\right)^{b}$ is a typical element of $\sqrt{G}$, then

$$
\frac{\dot{z}}{z}=\frac{a}{t}+\frac{b}{1+t+t^{2}}
$$

takes just four values

$$
0, \frac{1}{t}, \frac{1}{1+t+t^{2}}, \frac{(1+t)^{2}}{t\left(1+t+t^{2}\right)} .
$$

Since $\frac{\dot{y}}{y}-\frac{\dot{x}}{x}$ in $(4.2)$ is $\frac{\dot{z}}{z}$ for $z=\frac{y}{x}$, it follows that $x$ and $y$ are non-zero quotients of these. But the presence of the "stranger" $(1+t)^{2}$ means that the only possibilities for $x \neq 1$ and $y \neq 1$ are

$$
\frac{1+t+t^{2}}{t}, \quad \frac{t}{1+t+t^{2}}
$$

However then $x+y \neq 1$.

If $C x+C y$ has dimension 1 , then as $x+y=1$ we see that $x$ and $y$ lie in $C$. There is a biggest power $q$ of 2 with $x=x^{\prime q}$ and $y=y^{\prime q}$ for $x^{\prime}$ and $y^{\prime}$ not both in $C$. Now $x^{\prime}+y^{\prime}=1$ 
with $x^{\prime}$ and $y^{\prime}$ still in $\sqrt{G}$, and $C x^{\prime}+C y^{\prime}$ has dimension 2 ; but we have just seen this to be impossible. Thus the present lemma is proved.

To go further we welcome the above stranger into the bigger group $H$ with generators $t, 1+t+t^{2},(1+t)^{2}$. Here $\sqrt{H}$ has generators $t, 1+t+t^{2}, 1+t$.

Lemma 7. We have

$$
V_{2}(\sqrt{H})=\bigcup_{\pi \in S_{3}\left(\Pi_{2}\right)} \bigcup_{e=0}^{\infty} \pi^{2^{e}}
$$

for the set $\Pi_{2}$ consisting of

$$
\begin{gathered}
(1, t, 1+t), \quad\left(1, t(1+t), 1+t+t^{2}\right), \quad\left(1, t^{3},(1+t)\left(1+t+t^{2}\right)\right), \quad\left(1,(1+t)^{3}, t\left(1+t+t^{2}\right)\right), \\
\left(t,(1+t)^{2}, 1+t+t^{2}\right), \quad\left(t^{2}, 1+t, 1+t+t^{2}\right), \quad\left(t^{3},(1+t)^{3}, 1+t+t^{2}\right) .
\end{gathered}
$$

Proof. Again it suffices to consider $x$ and $y$ in $\sqrt{H}$ with $x+y=1$.

Assume first that $C x+C y$ has dimension 2. Now if $z=t^{a}\left(1+t+t^{2}\right)^{b}(1+t)^{c}$ is a typical element of $\sqrt{H}$, then

$$
\frac{\dot{z}}{z}=\frac{a}{t}+\frac{b}{1+t+t^{2}}+\frac{c}{1+t}
$$

These are the elements in (4.3) together with their sums with $1 /(1+t)$; that is,

$$
\frac{1}{1+t}, \quad \frac{1}{t(1+t)}, \quad \frac{t^{2}}{(1+t)\left(1+t+t^{2}\right)}, \quad \frac{1}{t(1+t)\left(1+t+t^{2}\right)} .
$$

Therefore $x$ and $y$ are non-zero quotients of elements of (4.3) and (4.4). This time we find no strangers; and in fact each of the possible 42 values for $x$ in $\sqrt{H}$ leads also to $y=1-x$ in $\sqrt{H}$. We verify without difficulty that the resulting 42 solutions $(x, y, 1)$ fall in 7 orbits under $S_{3}$ as stated in the present lemma. As in the proof of Lemma 6 , the case of dimension 1 supplies the exponents $2^{e}$, and this completes the proof.

Next we move to $V_{3}$. We define the torsion coset $Z_{01}$ in $V_{3}$ by $x_{0}=x_{1}, x_{2}=x_{3}$.

Lemma 8. We have

$$
V_{3}(\sqrt{G})=\bigcup_{Z \in S_{4}\left(Z_{01}\right)} Z(\sqrt{G}) \cup \bigcup_{\pi \in S_{4}\left(\Pi_{3}\right)} \bigcup_{e=0}^{\infty} \pi^{2^{e}}
$$


for the set $\Pi_{3}$ consisting of

$$
\begin{gathered}
\left(1, t, t^{2}, 1+t+t^{2}\right), \quad\left(1, t^{3}, t^{2}\left(1+t+t^{2}\right),\left(1+t+t^{2}\right)^{2}\right), \quad\left(1, t^{3}, 1+t+t^{2}, t\left(1+t+t^{2}\right)\right) \\
\left(t, t^{4}, 1+t+t^{2},\left(1+t+t^{2}\right)^{2}\right), \quad\left(1, t^{6}, t\left(1+t+t^{2}\right)^{2},\left(1+t+t^{2}\right)^{3}\right) .
\end{gathered}
$$

Proof. Take a point $\left(x_{0}, x_{1}, x_{2}, x_{3}\right)$ of $V_{3}(\sqrt{G})$ not in any $Z(\sqrt{G})$ in (4.5). Write $d$ for the dimension of $C x_{0}+C x_{1}+C x_{2}+C x_{3}$ over $C$. Since $[K: C]=2$ we have $d=1$ or $d=2$.

Assume first that $d=2$. We will prove that our point lies in $S_{4}\left(\Pi_{3}\right)$.

To this end define $i, j$ to be equivalent if $x_{i} / x_{j}$ lies in $C$. We show that at least one of the classes is a singleton. This is clear if the number $h$ of classes is 4 or even 3 . As $d=2$ we cannot have $h=1$. So assume $h=2$ and there is no singleton.

Now both classes must have two elements. But if say $x_{0} / x_{1}$ and $x_{2} / x_{3}$ lie in $C$, then neither quotient can be 1 and the identity

$$
\frac{x_{0}}{x_{3}}=\frac{1+\frac{x_{2}}{x_{3}}}{1+\frac{x_{1}}{x_{0}}}
$$

shows that $x_{0} / x_{3}$ lies in $C$. Thus $h=1$, a contradiction which shows that there must indeed be a singleton.

We can assume that this singleton consists of $x_{3}$. That means that

$$
y_{i}=\frac{\dot{x}_{i}}{x_{i}}-\frac{\dot{x}_{3}}{x_{3}} \neq 0 \quad(i=0,1,2)
$$

Further each $y_{i}$ is itself a logarithmic derivative of something in $\sqrt{G}$, and so it lies in a finite subset of $\sqrt{H}$ by (4.3). Also the equation

$$
y_{0} x_{0}+y_{1} x_{1}+y_{2} x_{2}=0
$$

follows from $x_{0}+x_{1}+x_{2}+x_{3}=0$ and its derivative. This remark is in fact a condensed version of the arguments of $[\mathrm{M}]$ (pp.198,199). Therefore we have a point of $V_{2}(\sqrt{H})$.

Thus there are $q=2^{e}$ and $\pi=\left(\xi_{0}, \xi_{1}, \xi_{2}\right)$ as in Lemma 7 such that

$$
\frac{y_{1} x_{1}}{y_{0} x_{0}}=\left(\frac{\xi_{1}}{\xi_{0}}\right)^{q}, \quad \frac{y_{2} x_{2}}{y_{0} x_{0}}=\left(\frac{\xi_{2}}{\xi_{0}}\right)^{q} \text {. }
$$


Already this leads to an algorithm for finding our point of $V_{3}(\sqrt{G})$. Namely for each $\pi$ we know from Lemma 6 that not both of $\xi_{1} / \xi_{0}, \xi_{2} / \xi_{0}$ lie in $\sqrt{G}$. So there is at most one $q$ such that

$$
\frac{x_{1}}{x_{0}}=\frac{y_{0}}{y_{1}}\left(\frac{\xi_{1}}{\xi_{0}}\right)^{q}, \quad \frac{x_{2}}{x_{0}}=\frac{y_{0}}{y_{2}}\left(\frac{\xi_{2}}{\xi_{0}}\right)^{q}
$$

both lie in $\sqrt{G}$. Further we can easily see by considering powers of $1+t$ that $q=1,2$. For example by Lemma 6 at least one of $\xi_{1} / \xi_{0}, \xi_{2} / \xi_{0}$ must involve $1+t$; but then by (4.3) a resulting $(1+t)^{4}$ could not be cancelled in (4.6). If there is such a $q$, then we need only check whether

$$
\frac{x_{3}}{x_{0}}=1+\frac{x_{1}}{x_{0}}+\frac{x_{2}}{x_{0}}
$$

also lies in $\sqrt{G}$. Here we are still allowed to permute $x_{0}, x_{1}, x_{2}$ and so we can use the symmetry to reduce the work by factor of six.

The case $d=1$ is dealt with as in the proof of Lemma 6 by reducing to $x+y+z=1$ and using $x=x^{\prime q}, y=y^{\prime q}$ and now $z=z^{\prime q}$.

All this means that the left-hand side of (4.5) is contained in the right-hand side. As the converse assertion is quickly checked, this completes the proof.

Now thanks to the one-to-one assertion in Lemma 5 we can find all the non-mixing sets of size 4. As $\Pi_{3}$ has five pre-broad elements, all in different proportionality classes, there are five equivalence classes. We can identify generators $v_{1}, v_{2}$ of $\sqrt{G}$ with $t, 1+t+t^{2}$ respectively (so that $\mathbf{Q}=\left(\begin{array}{ll}1 & 0 \\ 0 & 3\end{array}\right)$ in the proof). We find the integral representatives

$$
\begin{aligned}
& \{(0,0),(3,0),(6,0),(0,1)\} \\
& \{(0,0),(9,0),(6,1),(0,2)\} \\
& \{(0,0),(9,0),(0,1),(3,1)\} \\
& \{(3,0),(12,0),(0,1),(0,2)\} \\
& \{(0,0),(18,0),(3,2),(0,3)\}
\end{aligned}
$$

the first of which appears in $[S]$ (p.278) and (4.1) above.

5. Another example. Here we deal with a non-principal ideal, of which there are no examples in the mixing Chapter 28 of [S]. It is the $\mathcal{P}$ generated by 2 and $(1.5)$ in $\mathcal{R}=$ 
$\mathbf{Z}\left[u_{1}, u_{1}^{-1}, u_{2}, u_{2}^{-1}, u_{3}, u_{3}^{-1}\right]$. Each of the displayed generators shows that the corresponding $\alpha$ is not 4 -mixing by providing the non-mixing sets

$$
\{(0,0,0),(1,0,0),(2,0,0),(0,1,0)\}, \quad\{(0,0,0),(1,0,0),(3,0,0),(0,0,1)\} .
$$

We will prove here that $\alpha$ is 3 -mixing and that there are exactly 134 equivalence classes for non-mixing sets of size 4 .

The group $G$ has generators the images of $u_{1}, u_{2}, u_{3}$ in the quotient field $K$ of $\mathcal{R} / \mathcal{P}$. We may identify $K$ with $\mathbf{F}_{2}(t)$ and the generators with $t, 1+t+t^{2}, 1+t+t^{3}$ respectively. As these irreducible polynomials are clearly multiplicatively independent, we see already that $\alpha$ is 2-mixing. It is easy to see that $G=\sqrt{G}$. To go further we need again the field $C=\mathbf{F}_{2}\left(t^{2}\right)$ of differential constants.

Already by Lemma 5 above with $n=2$, the next lemma shows that $\alpha$ is 3 -mixing.

Lemma 9. The set $V_{2}(\sqrt{G})$ is empty.

Proof. It suffices to deduce a contradiction from the existence of $x$ and $y$ in $\sqrt{G}$ with $x+y=1$.

Assume first that the $C$-vector space $C x+C y$ has dimension 2. We get again (4.2). Now if $z=t^{a}\left(1+t+t^{2}\right)^{b}\left(1+t+t^{3}\right)^{c}$ is a typical element of $\sqrt{G}$, then

$$
\frac{\dot{z}}{z}=\frac{a}{t}+\frac{b}{1+t+t^{2}}+\frac{c\left(1+t^{2}\right)}{1+t+t^{3}}
$$

takes eight values, which are

$$
0, \frac{1}{t}, \frac{1}{1+t+t^{2}}, \frac{(1+t)^{2}}{t\left(1+t+t^{2}\right)}
$$

as in (4.3) together with

$$
\frac{(1+t)^{2}}{1+t+t^{3}}, \quad \frac{1}{t\left(1+t+t^{3}\right)}, \quad \frac{t^{4}}{\left(1+t+t^{2}\right)\left(1+t+t^{3}\right)}, \quad \frac{(1+t)^{4}}{t\left(1+t+t^{2}\right)\left(1+t+t^{3}\right)} .
$$

The presence of strangers leads now to 14 possibilities for quotients $x \neq 0,1$ in $\sqrt{G}$. However it is quickly checked that then $y=1+x$ is not among them.

The case of dimension 1 follows just as in the proof of Lemma 6 . 
To go further we need the bigger group $H$ with generators $t, 1+t+t^{2}, 1+t+t^{3},(1+t)^{2}$. Here $\sqrt{H}$ has generators $t, 1+t+t^{2}, 1+t+t^{3}, 1+t$. For an element $\mathbf{e}=(a, b, c, d)$ of $\mathbf{F}_{2}^{4}$ we write

$$
P(\mathbf{e})=t\left(1+t+t^{2}\right)\left(1+t+t^{3}\right)(1+t)\left(\frac{a}{t}+\frac{b}{1+t+t^{2}}+\frac{c\left(1+t^{2}\right)}{1+t+t^{3}}+\frac{d}{1+t}\right)
$$

in $\mathbf{F}_{2}[t]$. Write $\mathbf{0}=(0,0,0,0)$ and $\mathbf{1}=(1,1,1,1)$.

Lemma 10. We have

$$
V_{2}(\sqrt{H})=\bigcup_{\pi \in \Pi} \bigcup_{e=0}^{\infty} \pi^{2^{e}}
$$

for the set $\Pi$ consisting of the 168 elements $\left(P\left(\mathbf{e}_{0}\right), P\left(\mathbf{e}_{1}\right), P\left(\mathbf{e}_{2}\right)\right)$ with

$$
\mathbf{e}_{0}+\mathbf{e}_{1}+\mathbf{e}_{2}=\mathbf{0}, \quad \mathbf{e}_{0} \neq \mathbf{0}, \mathbf{1}, \mathbf{e}_{1} \neq \mathbf{0}, \mathbf{1}, \mathbf{e}_{2} \neq \mathbf{0}, \mathbf{1}
$$

Proof. Now if $z=t^{a}\left(1+t+t^{2}\right)^{b}\left(1+t+t^{3}\right)^{c}(1+t)^{d}$ is a typical element of $\sqrt{H}$, then

$$
\frac{\dot{z}}{z}=\frac{a}{t}+\frac{b}{1+t+t^{2}}+\frac{c\left(1+t^{2}\right)}{1+t+t^{3}}+\frac{d}{1+t}
$$

as in (5.4). And (4.2) shows that projectively $(x, y, 1)$ is $(\dot{y} / y,-\dot{x} / x, \dot{y} / y-\dot{x} / x)$, so after multiplication of all coordinates by $t(1+t)\left(1+t+t^{2}\right)\left(1+t+t^{3}\right)$ we get $\pi=$ $\left(P\left(\mathbf{e}_{0}\right), P\left(\mathbf{e}_{1}\right), P\left(\mathbf{e}_{2}\right)\right)$ with $\mathbf{e}_{0}+\mathbf{e}_{1}+\mathbf{e}_{2}=\mathbf{0}$. We certainly have to avoid $P(\mathbf{0})=0$, but all other $P(\mathbf{e})$ turn out to be in $\sqrt{H}$ with the single exception of $P(\mathbf{1})=\left(1+t^{2}+t^{3}\right)^{2}$. Then we check that the resulting $\pi$ are all different (however that is not crucial to the rest of the argument). This completes the proof.

For the move to $V_{3}$ we use as above the torsion coset $Z_{01}$ defined by $x_{0}=x_{1}, x_{2}=x_{3}$. But if we had known the outcome we might have never started on this example.

Lemma 11. We have

$$
V_{3}(\sqrt{G})=\bigcup_{Z \in S_{4}\left(Z_{01}\right)} Z(\sqrt{G}) \cup \bigcup_{\pi \in S_{4}\left(\Pi_{3}\right)} \bigcup_{e=0}^{\infty} \pi^{2^{e}}
$$

for a set $\Pi_{3}$ consisting of 134 elements containing

$$
\left(1, t, t^{2}, 1+t+t^{2}\right), \quad\left(1, t, t^{3}, 1+t+t^{3}\right),
$$




$$
\left(t^{2}, t^{3}, 1+t+t^{2}, 1+t+t^{3}\right), \quad\left(t, 1+t+t^{2}, t\left(1+t+t^{2}\right), 1+t+t^{3}\right)
$$

through to the Baby Gremlin

$$
\left(t^{21}\left(1+t+t^{3}\right), t^{20}\left(1+t+t^{2}\right),\left(1+t+t^{2}\right)^{12},\left(1+t+t^{3}\right)^{4}\right)
$$

and the Gremlin

$$
\left(t^{25}, t^{20}\left(1+t+t^{2}\right)\left(1+t+t^{3}\right),\left(1+t+t^{2}\right)^{12},\left(1+t+t^{3}\right)^{4}\right)
$$

Proof. As in the proof of Lemma 8 we take a point $\left(x_{0}, x_{1}, x_{2}, x_{3}\right)$ of $V_{3}(\sqrt{G})$ not in any $Z(\sqrt{G})$ with $d=1$ or $d=2$ for the dimension of $C x_{0}+C x_{1}+C x_{2}+C x_{3}$ over $C$.

Assume first that $d=2$. Just as in the proof of Lemma 8 we can assume that

$$
y_{i}=\frac{\dot{x}_{i}}{x_{i}}-\frac{\dot{x}_{3}}{x_{3}} \neq 0 \quad(i=0,1,2)
$$

Further each $y_{i}$ is itself a logarithmic derivative of something in $\sqrt{G}$, and so it lies in a finite subset of $\sqrt{H}$ by (5.2) and (5.3). We also get the equation

$$
y_{0} x_{0}+y_{1} x_{1}+y_{2} x_{2}=0
$$

and so a point of $V_{2}(\sqrt{H})$.

Thus there are $q=2^{e}$ and $\mathbf{e}_{0}, \mathbf{e}_{1}, \mathbf{e}_{2}$ satisfying the conditions of Lemma 10 such that

$$
\frac{y_{1} x_{1}}{y_{0} x_{0}}=\left(\frac{P\left(\mathbf{e}_{1}\right)}{P\left(\mathbf{e}_{0}\right)}\right)^{q}, \quad \frac{y_{2} x_{2}}{y_{0} x_{0}}=\left(\frac{P\left(\mathbf{e}_{2}\right)}{P\left(\mathbf{e}_{0}\right)}\right)^{q} .
$$

Already this leads to an algorithm for finding our point of $V_{3}(\sqrt{G})$. Namely for each $\mathbf{e}_{0}, \mathbf{e}_{1}, \mathbf{e}_{2}$ we know from Lemma 9 that not both of $P\left(\mathbf{e}_{1}\right) / P\left(\mathbf{e}_{0}\right), P\left(\mathbf{e}_{2}\right) / P\left(\mathbf{e}_{0}\right)$ lie in $\sqrt{G}$. So there is at most one $q$ such that

$$
\frac{x_{1}}{x_{0}}=\frac{y_{0}}{y_{1}}\left(\frac{P\left(\mathbf{e}_{1}\right)}{P\left(\mathbf{e}_{0}\right)}\right)^{q}, \quad \frac{x_{2}}{x_{0}}=\frac{y_{0}}{y_{2}}\left(\frac{P\left(\mathbf{e}_{2}\right)}{P\left(\mathbf{e}_{0}\right)}\right)^{q}
$$

both lie in $\sqrt{G}$. Further we can easily see that $q=1,2,4$. For example by Lemma 9 at least one of $P\left(\mathbf{e}_{1}\right) / P\left(\mathbf{e}_{0}\right), P\left(\mathbf{e}_{2}\right) / P\left(\mathbf{e}_{0}\right)$ must involve $1+t$; but then by (5.2) and (5.3) a 
resulting $(1+t)^{8}$ could not be cancelled in (5.5). If there is such a $q$, then we need only check whether

$$
\frac{x_{3}}{x_{0}}=1+\frac{x_{1}}{x_{0}}+\frac{x_{2}}{x_{0}}
$$

also lies in $\sqrt{G}$. This was originally carried out in 2010 by means of an interactive procedure on Maple. With mounting horror we realized that the many solutions were not obligingly organizing themselves into a few classes. After 20 hours we drew up a list of representative solutions numbered from 1 to the Eddingtonian 137. But Alexandre Warin in his 2012 Master Thesis observed that seven solutions appeared twice, then found four more solutions, and showed that there are no others involving exponents at most 21. Finally in 2016 the interactive procedure was repeated more carefully over 60 hours to show that Warin's list is indeed complete. The resulting 134 is just about Beethovenian.

The case $d=1$ is dealt with as in the proof of Lemma 8 using $x=x^{\prime q}, y=y^{\prime q}, z=z^{\prime q}$. This completes the present proof.

Again thanks to the one-to-one assertion in Lemma 5 we can find all the non-mixing sets of size 4. It is quickly checked that every $\pi$ in $\Pi_{3}$ is pre-broad. Also the corresponding non-mixing set $M=\left\{\mathbf{m}_{0}, \mathbf{m}_{1}, \mathbf{m}_{2}, \mathbf{m}_{3}\right\}$ turns up naturally in semi-reduced form. Furthermore the coordinates of $\mathbf{m}_{0}, \mathbf{m}_{1}, \mathbf{m}_{2}, \mathbf{m}_{3}$ are all coprime and so $M$ is reduced. So we have the unique representative, and we only have to check that they are all different (itself not entirely painless).

Two $\pi$ come immediately from the generators, namely the polynomials

$$
1+u_{1}+u_{1}^{2}+u_{2}, \quad 1+u_{1}+u_{1}^{3}+u_{3}
$$

with respective non-mixing sets

$$
\{(0,0,0),(1,0,0),(2,0,0),(0,1,0)\}, \quad\{(0,0,0),(1,0,0),(3,0,0),(0,0,1)\}
$$

as in (5.1).

The next two simplest polynomials are perhaps

$$
u_{1}^{2}+u_{1}^{3}+u_{2}+u_{3}, \quad u_{1}+u_{2}+u_{3}+u_{1} u_{2}
$$

with

$$
\{(2,0,0),(3,0,0),(0,1,0),(0,0,1)\}, \quad\{(1,0,0),(0,1,0),(0,0,1),(1,1,0)\}
$$


The two most complicated are

$$
u_{1}^{21} u_{3}+u_{1}^{20} u_{2}+u_{2}^{12}+u_{3}^{4}, \quad u_{1}^{25}+u_{1}^{20} u_{2} u_{3}+u_{2}^{12}+u_{3}^{4}
$$

with

$$
\{(21,0,3),(20,1,0),(0,12,0),(0,0,4)\}, \quad\{(25,0,0),(20,1,1),(0,12,0),(0,0,4)\} .
$$

Acknowledgment. The first author was partially supported by NSF grant DMS-1601229.

\section{References}

[ABB] L. Arenas-Carmona, D. Berend and V. Bergelson, Ledrappier's system is almost mixing of all orders, Ergod. Th. and Dynam. Sys. 28 (2008), 339-365.

[BM] W.D. Brownawell and D. Masser, Vanishing sums in function fields, Math. Proc. Cambridge Phil. Soc. 100 (1986), 427-434.

[BMZ] E. Bombieri, D. Masser and U. Zannier, Anomalous subvarieties - structure theorems and applications, Int. Math. Research Notices 2007, Article ID rnm057 (33 pages), doi: 10.1093/imrn/rnm057.

[D] H. Derksen, A Skolem-Mahler-Lech theorem in positive characteristic and finite automata, Inventiones Math. 168 (2007), 175-224.

[DM1] H. Derksen and D. Masser, Linear equations over multiplicative groups, recurrences, and mixing I, Proc. London Math. Soc. 104 (2012), 1045-1083.

[DM2] H. Derksen and D. Masser, Linear equations over multiplicative groups, recurrences, and mixing II, Indagationes Math. 26 (2015), 113-136.

[Led] F. Ledrappier, Un champ markovien peut être d'entropie nulle et mélangeant, C.R. Acad. Sci. Paris 287 (1978), 561-563.

[Lei] D. Leitner, Linear equations over multiplicative groups in positive characteristic, Acta Arith. 153 (2012), 325-347.

[LM] T. Loher and D. Masser, Uniformly counting points of bounded height, Acta Arith. 111 (2004), 277-297. 
[M] D. Masser, Mixing and linear equations over groups in positive characteristic, Israel J. Math. 142 (2004), 189-204.

[SW] K. Schmidt and T. Ward, Mixing automorphisms of compact groups and a theorem of Schlickewei, Inventiones Math. 111 (1993), 69-76.

[S] K. Schmidt, Dynamical systems of algebraic origin, Birkhäuser, Basel 1995.

[SV] H. P. Schlickewei and C. Viola, Polynomials that divide many k-nomials, Number Theory in Progress (eds. K. Györy, H. Iwaniec, J. Urbanowicz), Walter de Gruyter 1999 (pp.445-450).

[W] T. Ward, Three results on mixing shapes, New York J. Math. 3A (1997), 1-10.

H. Derksen: Department of Mathematics, University of Michigan, East Hall 530 Church Street, Ann Arbor, Michigan 48104, U.S.A. (hderksen@umich.edu)

D. Masser: Departement Mathematik und Informatik, Universität Basel, Spiegelgasse 1, 4051 Basel, Switzerland (David.Masser@unibas.ch).

21.10.2016

revised 21.11.2016 


\section{LATEST PREPRINTS}

No. Author: Title

2016-06 ～M. Dambrine, I. Greff, H. Harbrecht, B. Puig

Numerical solution of the homogeneous Neumann boundary value problem on domains with a thin layer of random thickness

2016-07 G. Alberti, G. Crippa, A. L. Mazzucato

Exponential self-similar mixing by incompressible flows

2016-08 M. Bainbridge, P. Habegger, M. Möller

Teichmüller curves in genus three and just likely intersections in $G^{n}{ }_{m} \times G_{a}^{n}$

2016-09 Gabriel A. Dill

Effective approximation and Diophantine applications

2016-10 J. Blanc, S. Zimmermann

Topological simplicity of the Cremona groups

2016-11 I. Hedén, S. Zimmermann

The decomposition group of a line in the plane

2016-12 J. Ballani, D. Kressner, M. Peters

Multilevel tensor approximation of PDEs with random data

2016-13Ｍ. J. Grote, M. Kray, U. Nahum

Adaptive eigenspace method for inverse scattering problems in the frequency domain

2016-14 H. Harbrecht, M. Peters, M. Schmidlin

Uncertainty quantification for PDEs with anisotropic random diffusion

2016-15 F. Da Lio, L. Martinazzi

The nonlocal Liouville-type equation in $R$ and conformal immersions of the disk with boundary singularities

2016-16 A. Hyder

Conformally Euclidean metrics on $R^{n}$ with arbitrary total $Q$-curvature

2016-17 G. Mancini, L. Martinazzi

The Moser-Trudinger inequality and its extremals on a disk via energy estimates

2016-18 R. N. Gantner, M. D. Peters

Higher order quasi-Monte Carlo for Bayesian shape inversion

Preprints are available under https://math.unibas.ch/research/publications 


\section{LATEST PREPRINTS}

No. Author: Title

2016-19 C. Urech

Remarks on the degree growth of birational transformations

2016-20 S. Dahlke, H. Harbrecht, M. Utzinger, M. Weimar

Adaptive wavelet BEM for boundary integral equations: Theory and numerical experiments

2016-21 A. Hyder, S. Iula, L. Martinazzi

Large blow-up sets for the prescribed Q-curvature equation in the Euclidean space

2016-22 P. Habegger

The norm of Gaussian periods

2016-23 P. Habegger

Diophantine approximations on definable sets

2016-24 F. Amoroso, D. Masser

Lower bounds for the height in Galois extensions

2016-25 W. D. Brownawell, D. W. Masser

Zero estimates with moving targets

2016-26 H. Derksen, D. Masser

Linear equations over multiplicative groups, recurrences, and mixing III

Preprints are available under https://math.unibas.ch/research/publications 